\title{
Using visible light to tune boronic acid-ester equilibria
}

\author{
Joseph V. Accardo, Emily R. McClure, Martín A. Mosquera ${ }^{\dagger}$ and Julia A. Kalow* \\ Department of Chemistry, Northwestern University, Evanston, Illinois, 60208, United States
}

Supporting Information Placeholder

\begin{abstract}
We report a series of azobenzene boronic acids that reversibly control the extent of diol binding via photochemical isomerization. When the boronic acid is ortho to the azo group, the thermodynamically-favored $E$ isomer binds weakly with diols to form boronic esters. Isomerization of the $E$ azobenzene to its $Z$ isomer enhances diol binding, and the magnitude of this enhancement is affected by the azobenzene structure. 2,6-Dimethoxy azobenzene boronic acids show over 20-fold enhancement in binding upon $E$ $Z$ isomerization, which can be triggered with red light. Competition experiments and computational studies suggest that the changes in binding affinity originate from stabilization of the $E$ boronic acids and destabilization of the $E$ boronic esters. We demonstrate a correlation between diol binding and photostationary state: different wavelengths of irradiation yield different quantities of bound diol. Higher binding constants for the $Z$ isomer relative to the $E$ isomer was observed with all diols investigated, including cyclic diols, nitrocatechol, biologically relevant compounds, and polyols. This photoswitch was employed to "catch and release" a fluorescently tagged diol in buffered water. By tethering this photoswitch to a poly(ethylene glycol) star polymer, we can tune the stiffness of covalent adaptable hydrogels using different wavelengths of visible light. This work establishes photoswitchable equilibria as a tool for the reversible ligation of molecular and macromolecular species.
\end{abstract}

\section{INTRODUCTION}

Dynamic covalent chemistry (DCC) enables thermodynamically-driven reorganization of molecular and macromolecular systems. ${ }^{1}$ In the last 20 years, dynamic covalent chemistry has expanded both in utility and scope, with the most popular reactions including transesterification, transimination, Diels-Alder cycloaddition, conjugate addition-elimination, disulfide exchange, metathesis, and boronic ester exchange. ${ }^{2-3}$ The reversibility of these reactions enables applications in combinatorial library development, molecular recognition, self-healing polymers, 2- and 3-D covalent organic frameworks, and matrices for 3D cell-culture. ${ }^{2,4-9}$ With insight into the reaction mechanism, DCC equilibria can be adjusted by structural modification of exchange partners, providing materials with a wide range of properties. In stimuli-responsive materials, these equilibria are influenced by stimuli such as light, temperature, $\mathrm{pH}$, and concentration. ${ }^{10}$ Light represents an ideal stimulus because it can be applied at readily tuned wavelengths and fluxes with spatiotemporal precision. In applications where sample penetration depth and biocompatibility are of concern, irradiation in the red and near-infrared range is advantageous. ${ }^{11}$

To influence DCC with light, a photoresponsive substrate must be coupled to the exchange reaction. ${ }^{12-14}$ Photoswitches are a class of compounds that undergo bidirectional switching in response to light, resulting in a physical change in shape, charge, conjugation, dipole, or $\mathrm{p} K_{\mathrm{a}} \cdot{ }^{15-23}$ When a photoswitch is coupled to DCC, the state of the switch may influence the dynamic reaction. ${ }^{24}$ In 2006, Branda and coworkers realized the first example of photoswitchable DCC, demonstrating that the Diels-Alder cycloaddition between a diarylethene photoswitch and a dienophile could be turned on or off (gated) depending upon the isomerization of the photoswitch (Figure 1a). ${ }^{25}$ In the open isomer, the diarylethene can undergo a [4+2] cycloaddition with a dienophile. The product could be photoswitched via a $6 \pi$ electrocyclization to yield a closed "locked" isomer, which is unable to participate in the Diels-Alder equilibrium. This work was further developed by Hecht, who applied this reactivity in photoactivatable maleimide prodrugs and self-healing polymer networks. ${ }^{26-29}$ In these reports, the DCC is either on or off.

We envisioned an alternative approach that modulates the overall equilibrium of a dynamic covalent bond using different wavelengths of light (Figure 1b). In this case, the equilibrium can operate in both states of the photoswitch, but to different extents. This system will enable bidirectional tuning of the reversible bond between small molecules and polymers to our photoswitch. In a complementary study, Hecht and coworkers investigated the effect of photoswitches on the rate of formation of imine dynamic covalent bonds, but did not study the photoswitches' influence on the bond equilibrium. ${ }^{30}$ We identified boronic esters as an ideal dynamic covalent bond for this purpose because the exchange occurs at room temperature with many different diol structures and is compatible with aqueous environments (Figure 1c). These attributes have found utility in molecular sensors, stress-relaxing hydrogels, and recyclable thermosets. ${ }^{31-39}$ Previous work has shown that the boronic acid-ester equilibrium is highly sensitive to the boronic acid structure. ${ }^{40-44}$ We anticipated that photoswitch isomerization could provide the reversible structural change capable of influencing this equilibrium. 
a) On-off (gated) control of the Diels-Alder reaction (Branda, 2006)

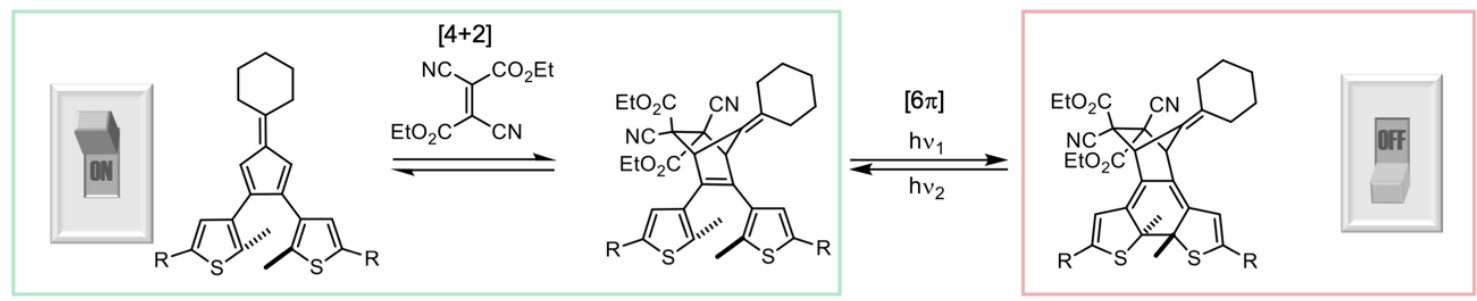

b) Our approach: equilibria tuned over a range using visible light

c) Dynamic covalent boronic acid-ester reaction
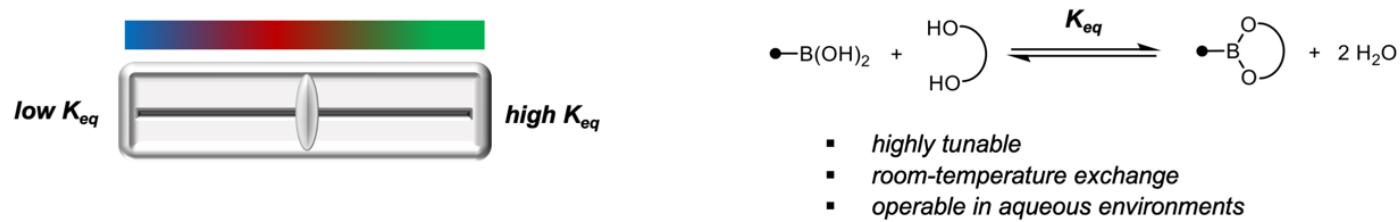

Figure 1. (a) The [4+2] cycloaddition between a diarylethene and dieneophile can be turned on or off via a $6 \pi$ electrocylization. (b) In this work, different wavelengths of irradiation tune the extent of an equilibrium. (c) The reversible reaction of boronic acids and diols forms boronic esters.

a)

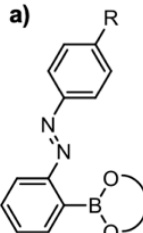

$\mathrm{R}=\mathrm{CO}_{2} \mathrm{Me}$
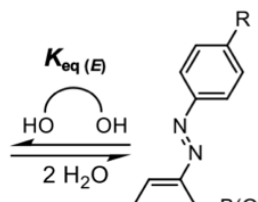

$\overbrace{-\mathrm{B}(\mathrm{OH})_{2}}^{\mathrm{N}}$
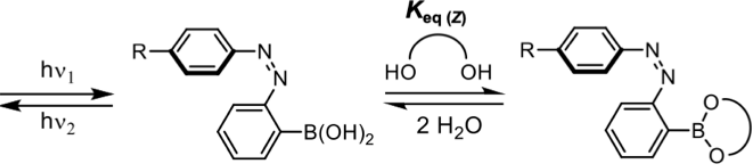

b)
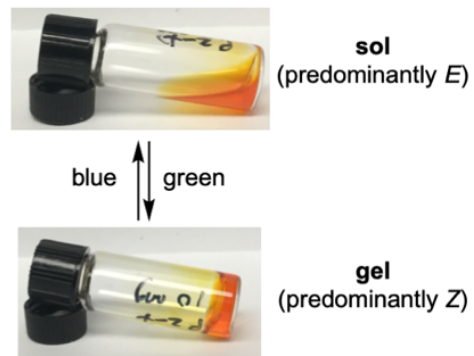

ge

(predominantly Z)

Figure 2. (a) The equilibrium between ortho-substituted azobenzene boronic acids and diols is influenced by the isomerization of the photoswitch, with the $Z$ isomer displaying a higher binding affinity with diols than the $E$ isomer. When the diol is pinacol, the $Z$ binding constant is 4 times greater than the $E$ binding constant. (b) Reversible sol-gel transitions of poly(ethylene glycol) hydrogels crosslinked with azobenzene boronic esters mediated by green and blue light (10 w/v\%, $0.1 \mathrm{M}$ PBS, $\mathrm{pH} 7.5)$.

Towards this goal, we synthesized ortho-substituted azobenzene boronic acids (Figure 2a). ${ }^{45-46}$ As azobenzenes undergo substantial changes in structure upon $E \rightarrow Z$ isomerization, we hypothesized that the boronic acid-ester equilibrium could be affected by the photoswitch state. Accordingly, we discovered that these compounds have isomerism-dependent equilibria with diols, wherein the $Z$ isomer has a higher binding affinity than the $E$ isomer $\left(K_{\text {eq( }}(Z)\right.$ $>K_{\mathrm{eq}(E)}$, Figure $\left.2 \mathrm{a}\right) .{ }^{47}$ When incorporated into poly(ethylene glycol) networks, this preferential binding translated to reversible sol-gel transitions upon $E \rightarrow Z$ photoisomerization (Figure 2b). Interestingly, this dramatic physical change arose from a relatively modest difference in binding affinity to diols ( $4: 1$ for pinacol). We thus sought structural modifications of the azobenzene boronic acid that could increase the difference in binding affinity between the $E$ and $Z$ isomers, allowing us to expand the tunability of this photocontrolled dynamic covalent bond and its potential applications.

In this report, we demonstrate that simple modifications of the azobenzene boronic acid allow us to tune the relative binding affinity of $Z$ vs. $E$ azobenzene boronic acids to diols, $K_{\text {rel }}=$ $K_{\text {eq }(Z)} / K_{\text {eq }(E)}$, from 2.4 to over 20 . Experimental results and computed structures suggest that the differences in $K_{\text {rel }}$ between a series of azobenzene boronic acids are due to both the stabilization of the $E$ boronic acids and the destabilization of the $E$ boronic esters, which can be tuned by azobenzene substitution. Our optimized azobenzene boronic acid can be photoswitched with red light and the $Z$ isomer has a long thermal half-life. Using the azobenzene boronic acid with the largest $K_{\text {rel }}$, the equilibrium between boronic acid and boronic ester can be tuned with different wavelengths of light. We demonstrate that this azobenzene boronic acid can "catch and release" a fluorescently tagged polyol in aqueous buffer. Lastly, we reversibly tune the stiffness of a covalent adaptable hydrogel with several wavelengths of visible light, including red light.

\section{RESULTS AND DISCUSSION}

\section{Ortho substitution increases the difference in binding affinity between $E$ and $Z$ isomers}

The equilibrium between boronic acids and diols strongly depends on the concentration of water and diol. Therefore, we designed ${ }^{1} \mathrm{H}$ and ${ }^{19} \mathrm{~F}$ NMR equilibrium competition binding experiments to compare the relative binding affinity between $E$ and $Z$ isomers in the same NMR tube, ensuring that the concentration of water and diol are the same for both isomers (Figure 3a; see Supporting Information (SI), section IV for details and derivation). Our investigations commenced with compound $\mathbf{1}$, a para-tolyl azobenzene boronic acid (Figure 3b). The para-methyl substituent was 


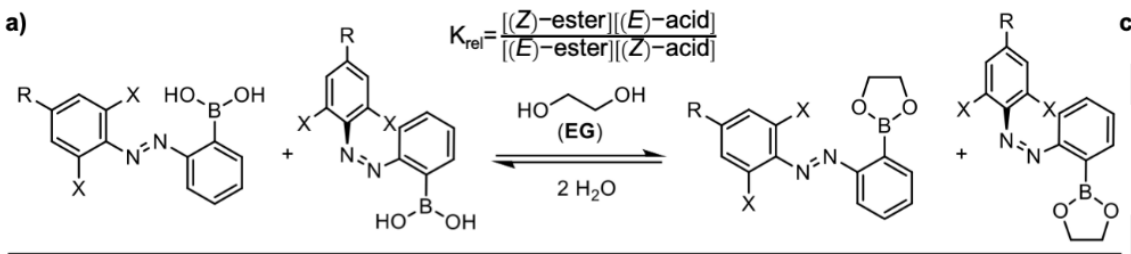

c)

b)<smiles>Cc1ccc(N=Nc2ccccc2O)cc1</smiles>

1

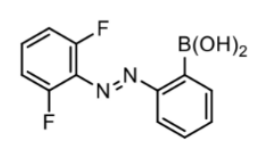

2

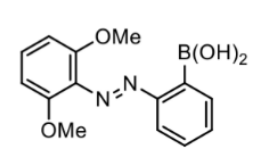

3

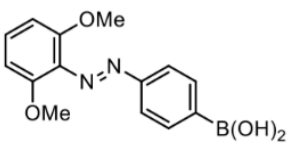

Figure 3. (a) Equilibrium competition binding experiment to compare the relative equilibrium binding constants of $E$ and $Z$ azobenzene boronic acids with diol. (b) Library of photoswitches synthesized and subjected to the above competition binding experiment. (c) Competition binding experiment results in DMSO- $d_{6}$ at $25^{\circ} \mathrm{C}$, thermal half-lives (days) and photostationary states $(\% Z)$ of azobenzene boronic-acids.

used in place of the phenyl analog, which exhibits overlapping ${ }^{1} \mathrm{H}$ NMR signals for the $E$ and $Z$ isomers. When a mixture of $(E)-\mathbf{1}$ and (Z)-1 was treated with excess ethylene glycol (EG) and $\mathrm{D}_{2} \mathrm{O}$ in DMSO- $d_{6}$ and allowed to equilibrate for 12 hours, we observed that $(Z)-\mathbf{1}$ binds EG approximately 2.4 times better than $(E)-\mathbf{1}(\mathrm{Krel}=$ 2.4) (Figure S8).

For our design of photoswitchable equilibria, the ideal photoswitch should feature visible-light bidirectional photoswitching, high photostationary states, and a long thermal half-life for the $Z$ isomer. Unfortunately, in addition to a modest difference between $E$ and $Z$ binding affinities, compound 1 only reached an appreciable photostationary state (PSS, 48\% Z) with ultraviolet (UV) irradiation at $365 \mathrm{~nm}$ (Figure 3c). By measuring the rate of thermal relaxation of $(Z)-\mathbf{1}$ to $(E)-\mathbf{1}$ at different temperatures and fitting to an Arrhenius plot (Figures S4), we could extrapolate the room-temperature $\left(25^{\circ} \mathrm{C}\right)$ half-life to be 3.5 days. Previous work has established that electronic and steric modifications of the azobenzene can alter key photochemical properties, which has been summarized well by Bandara and Burdette. ${ }^{16} \mathrm{We}$ anticipated that these modifications could simultaneously alter the equilibrium of the dynamic covalent bond. First, we sought to optimize the photochemical properties to achieve bidirectional visible-light photoswitching. Secondly, we aimed to increase the thermal stability of the $Z$ isomers.

A common strategy to enable azobenzene isomerization with visible light is substituting the aryl rings with resonance electrondonating and -withdrawing groups, creating "push-pull" azobenzenes. These substitution patterns red-shift the $\pi \rightarrow \pi^{*}$ of the $E$ isomer through a charge-transfer mechanism. Unfortunately, the $Z$ isomers tend to undergo rapid thermal isomerization ( $<$ seconds), due to a low activation barrier in the ground state, ${ }^{48}$ which would occur much faster than the dynamic covalent reaction. Hecht, Woolley, and others have installed halides or methyl ethers at the ortho positions, which separates the $n \rightarrow \pi^{*}$ absorption bands of the $E$ and $Z$ isomers and enables visible-light $E \rightarrow Z$ photoisomerization with enhanced $Z$ thermal stability. ${ }^{49-55}$ Inspired by this work, we synthesized ortho-difluoro-substituted azobenzene $\mathbf{2}$ and dimethoxy analog 3 to access visible-light photoswitching (Figure 3b). 2 exhibited a higher PSS $(67 \%$ Z) with green light $(525 \mathrm{~nm})$ irradiation and a dramatically enhanced thermal half-life relative to $1\left(\tau_{1 / 2}=70\right.$ days, Figure S5). The relative binding affinity, as determined by the equilibrium competition binding experiment with $\mathrm{EG}$, increased by a factor of 1.8 relative to 1 , to $K_{\text {rel }}=4.2$ (Figure 3c, Figure S9). When the fluorine atoms were replaced with methyl ethers in compound 3, photoswitching with red LEDs at $626 \mathrm{~nm}$ yielded PSS up to $54 \%$, albeit with a modest decrease in thermal half-life $\left(\tau_{1 / 2}=24\right.$ days, Figure S6). The competition experiment for compound $\mathbf{3}$ also revealed a dramatic increase in relative binding, where the $Z$ isomer binds $E G$ with over an order of magnitude higher affinity than the $E$ isomer $\left(K_{\text {rel }}=21.5\right.$, Figure $\left.\mathrm{S} 10\right)$. Figure 4 shows the ${ }^{1} \mathrm{H}$ NMR spectrum for the competition experiment of compound $\mathbf{3}$ with EG, in which $(Z)-\mathbf{3}$ is predominantly bound, while $(E)-\mathbf{3}$ is mainly unbound.

As a control, we synthesized an azobenzene derivative with the boronic acid at the 4' (para) position (compound 4). This compound exhibited little difference in binding affinities between the $E$ and $Z$ isomers $\left(K_{\text {rel }}=1.1\right.$, Figure $\left.\mathrm{S} 12\right)$. Thus, the proximity of the boronic acid to the phenylazo group is essential for the isomerismdependent changes in binding affinity.

\section{Ortho substitution influences the esterification equilibrium of the $E$ isomers}

We next set out to understand the origin of the dramatic increase in $K_{\text {rel }}$ for compound $\mathbf{3}$ compared to compounds $\mathbf{1}$ and $\mathbf{2}$. As $K_{\text {rel }}$ is defined as the ratio of $K_{\mathrm{eq}(Z)}$ to $K_{\mathrm{eq}(E)}$, this trend could originate in an increase in $K_{\mathrm{eq}(Z)}$, a decrease in $K_{\mathrm{eq}(E)}$, or a combination of both effects. We performed an equilibrium competition binding experiment to compare $(E) \mathbf{- 1}$ and $(E)$-2, and a separate experiment comparing $(E)-\mathbf{2}$ and (E)-3 (Figure S13). The $E$ isomers for 1-3 displayed significantly different binding affinities for EG, following
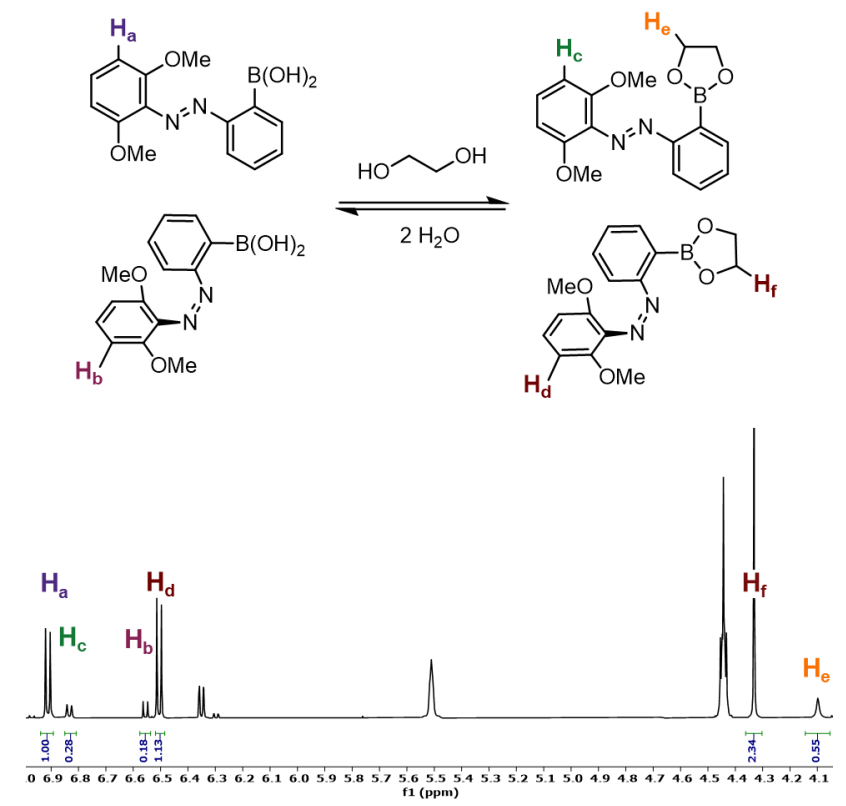

Figure 4. ${ }^{1} \mathrm{H}$ NMR of 3 and EG subjected to equilibrium competition binding conditions in DMSO- $d_{6}$. 
the $K_{\text {eq }}$ trend $(E)-\mathbf{3}<<(E)-\mathbf{2}<(E)-\mathbf{1}$, consistent with the overall changes in $K_{\text {rel }}$. In contrast, equilibrium competition experiments for the $Z$ isomers of $\mathbf{1}-\mathbf{3}$ revealed similar binding affinities to EG (Figure S14). Therefore, we concluded that the changes in $K_{\mathrm{eq}(E)}$, and not $K_{\text {eq(Z) }}$, lead to the observed differences in $K_{\text {rel }}$ between compounds 1-3.

Calculated structures and control experiments suggest a combination of reactant stabilization and product destabilization disfavors $E$ esterification

Why do $E$ isomers of azobenzene boronic acids consistently display lower binding affinities for diols than their corresponding $Z$ isomers? Why does $(E)-\mathbf{3}$ bind EG significantly worse than $(E)-\mathbf{2}$ and $(E)-1$ ? Structural changes that result in the stabilization of the $(E)$-acid, destabilization of destabilization of the $(E)$-ester, or a combination will correspond to less negative $\Delta G$ and decreased diol binding affinity (Figure $5 \mathrm{a}-\mathrm{b}$ ). We hypothesized that there are two main structural parameters influencing the degree of conjugation through the system and therefore the energy of the azobenzene boronic acid and ester. The first is the planarity of the azobenzene, which is described by the dihedral angle between the aryl rings and diazo (CCNN, $\Phi)$ (Figure 5c). For unsubstituted azobenzene, the energy is lowest when this angle is $0^{\circ}$ and highest at $90^{\circ}$ (Figure S29). The second parameter is the coplanarity of the azobenzene and the boronic acid or ester, which can be described by the CCBO dihedral angle $\psi$. This parameter correlates with the degree of conjugation between the boronic acid or ester with the adjacent aryl group (Figure 5c). Previous reports suggest that arylboronic acids and esters are stabilized when the non-bonding oxygen lone pairs and vacant $p$ orbital are in conjugation with the $\pi$-system of the aryl group, with a CCBO dihedral angle of $0^{\circ}$. We performed geometry scans of phenylboronic acid and the corresponding ester, confirming an energetic maximum at $90^{\circ}$ and a minimum at $0^{\circ}$, when conjugation is enhanced. (Figure S29).

To understand which scenario might be responsible for the decrease in $K_{\mathrm{eq}}(E)$ from $\mathbf{1}>\mathbf{2}>\mathbf{>}$, we calculated the optimized structures of $(E)-\mathbf{1}, \mathbf{2}$, and $\mathbf{3}$ in their bound and unbound forms with the $\mathrm{B} 3 \mathrm{LYP} / 6-31+\mathrm{G}^{* *}$ level of theory and performed frequency calculations to determine the lowest-energy rotamers. We modeled the boronic acids and esters in their trigonal planar form based on ${ }^{11} \mathrm{~B}$ NMR studies (Figure S11). We first examined the geometry-optimized structures of $(E)-\mathbf{1}$ through $(E)-\mathbf{3}$ in their unbound forms (Figure 6a). Each azobenzene is nearly planar, and the two CCNN dihedrals angles $\Phi\left(\mathrm{Ar}^{1}\right)$ and $\Phi\left(\mathrm{Ar}^{2}\right)$ are between $11^{\circ}$ and $17^{\circ}$ for a) Stabilization of $E$ acid

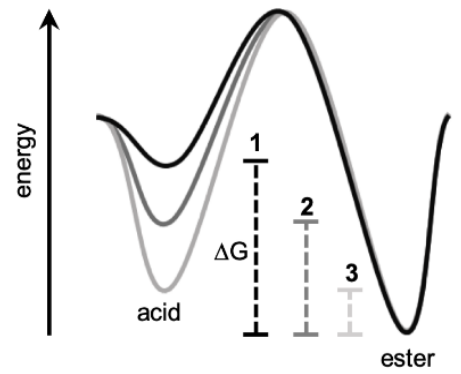

b) Destabilization of $E$ ester

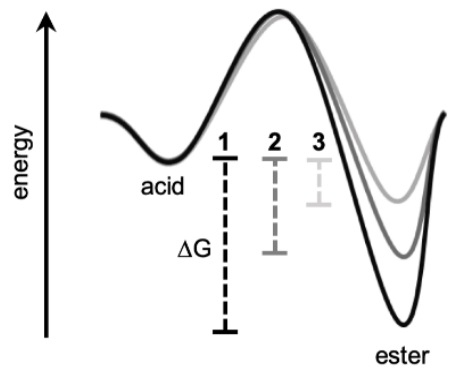

c) Key dihedral angles investigated

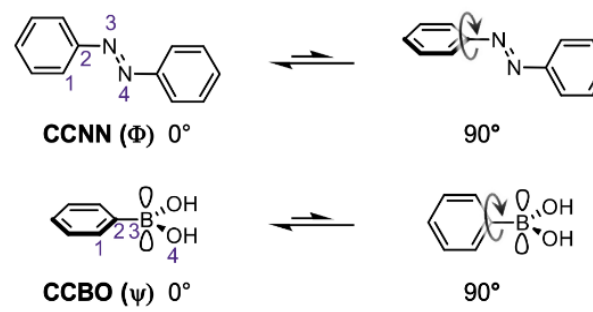

Figure 5. $(a, b)$ Energy diagrams portraying two potential origins of the observed trend in Keq, $(E)-\mathbf{1}>(E)-\mathbf{2}>(E)-\mathbf{3}$. (c) Key bond rotations that can influence the energy of azobenzene boronic acids and esters. a) Calculated structures of unbound azobenzene boronic acids $(E)-1$ through $(E)-3$
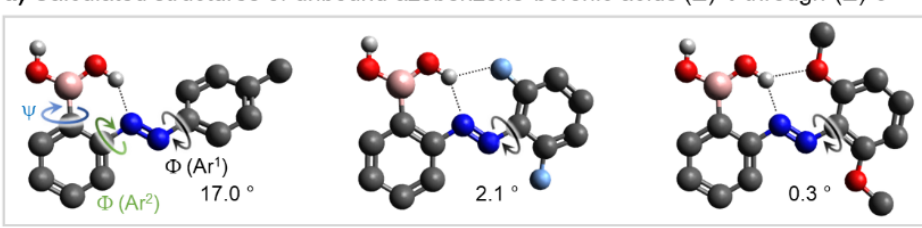

c) Calculated structures of bound azobenzene boronic esters $(E)-1$ through $(E)-3$

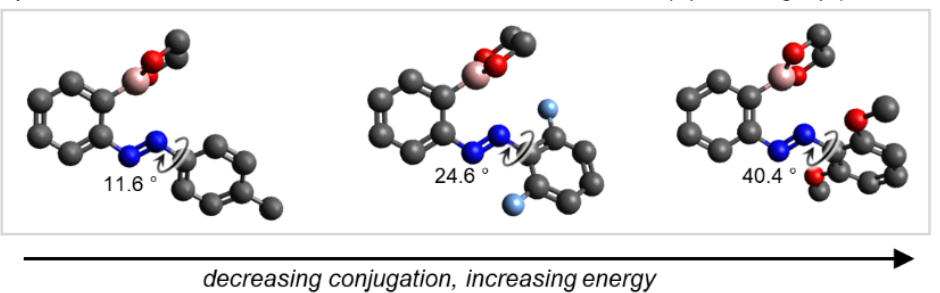

b) X-ray crystal structure of compound $(E)-1$ though $(E)-3$
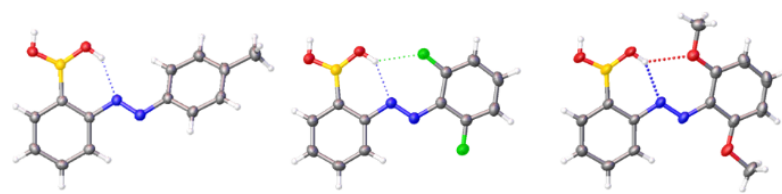

d) Control compounds 5-7
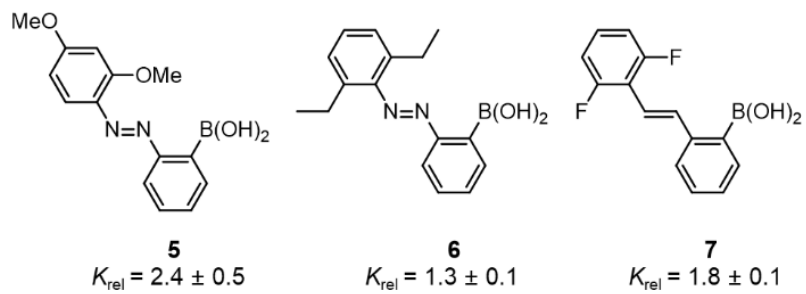

Figure 6. (a) Energy-minimized structures of boronic acids $(E)-\mathbf{1}$ through $(E)-3 . \Phi\left(\mathrm{Ar}^{1}\right.$, black), $\Phi\left(\mathrm{Ar}^{2}\right.$, green) and $\psi$ (blue) represent the two CCNN and one CCBO dihedral angles investigated, respectively. (b) Crystal structure of compound 1- 3 showing intramolecular hydrogen bonds. (c) Energy-minimized structures of boronic esters $(E)-\mathbf{1}$ through $(E)$-3. (d) Structure of control comounds 5-7 and their experimentally determined $K_{\text {rel }}$ values. 
$(E)-\mathbf{1}$ and between $0^{\circ}$ and $2^{\circ}$ for $(E)-\mathbf{2}$ and $(E)-\mathbf{3}$. The CCBO dihedral angles $\psi$ are close to $0^{\circ}$ for $\mathbf{1}-\mathbf{3}$, and an intramolecular hydrogen bond is present between the boronic acid proton and the proximal azobenzene nitrogen.

The boronic acid protons in the calculated structures of $(E)-2$ and (E)-3 also engage in H-bonding with one of the ortho-heteroatom substituents (fluorine or methoxy, respectively). This additional Hbonding interaction was confirmed in the single-crystal X-ray structures of $(E)-2$ and $(E)-3$ (Figure 6b). We hypothesize that the additional hydrogen bonds stabilize the boronic acid and lead to a less negative $\Delta G$ for esterification. While the $\mathrm{O}-\mathrm{H} \cdots \mathrm{F}-\mathrm{C}$ interaction in $\mathbf{2}$ is expected to be much weaker than with the methoxy group in 3 , the H-F distance of $2.27 \AA$ is consistent with previously reported $\mathrm{H}$-bonding interactions involving a C-F acceptor. ${ }^{56-57}$ In both 2 and $\mathbf{3}$, these additional hydrogen bonds assist in keeping the azobenzene nearly planar, whereas $(E)-\mathbf{1}$, which lacks H-bond acceptors at the ortho positions, is slightly more twisted. Again, these features were corroborated by the single-crystal X-ray structure of $(E)$ $\mathbf{1}$, in which $\Phi\left(\mathrm{Ar}^{1}\right)$ is $19.8^{\circ}$

Binding of EG to the $(E)$-azobenzene boronic acids is accompanied by varying degrees of twisting of the aryl rings and deviation of the CCNN dihedral angles from $0^{\circ}$. In the calculated structures, ortho-unsubstituted $(E)-1$ remains slightly twisted after binding EG $\left(11.6^{\circ}\right)$, while $(E)-2$ and $(E)-3$ display more significant twisting, exemplified by $\Phi\left(\mathrm{Ar}^{1}\right)$ angles of $24.6^{\circ}$ and $40.4^{\circ}$, respectively (Figure 6c, see Figure S31 for full list of angles). This twisting likely arises from repulsive steric interactions between the halide or methoxy ortho substituents and the boronic ester. For all compounds, we observe that the boronic ester is nearly perpendicular to the aryl ring $\left(75^{\circ} \leq \psi \leq 84^{\circ}\right)$, which avoids Van der Waals repulsion between the ester oxygen and distal nitrogen. We hypothesize that the large conformational changes engendered by diol binding reduce the degree of conjugation in the azobenzene and raise the energy of the boronic ester relative to the boronic acid. Since these conformational changes become more significant across the series $\mathbf{1}<\mathbf{2}$ $<\mathbf{3}, \Delta G$ becomes correspondingly less negative across this series, and the esterification becomes less favorable for $\mathbf{3}$ compared to $\mathbf{1}$ and 2. Alternative rotamers for the esters of $(E)$-1 through $(E)-\mathbf{3}$ (Figure S31) are less than $3 \mathrm{kcal} / \mathrm{mol}$ higher in energy but follow similar trends to those shown here, with the smallest conformational changes upon binding occurring for $(E)-1$ and the largest changes occurring for $(E)-\mathbf{3}$.

The optimized structures for the $Z$ isomers place the boronic acids away from the ortho substituents. Each boronic acid still displays an intramolecular hydrogen bond with the proximal nitrogen; however, there are no additional H-bonds with the ortho-fluoro or methoxy substituents in (Z)-2 and 3. The absence of steric interactions between the boronic esters and ortho substituents in the $Z$ isomers allows diol binding to occur with minimal structural rearrangement for compounds $(Z)-\mathbf{1}$ through $(Z)-\mathbf{3}$, as exemplified by small changes in $\Phi\left(\mathrm{Ar}^{1}\right), \Phi\left(\mathrm{Ar}^{2}\right)$, and $\psi$ upon diol binding (Figure 7 for 3; see Figure S32 for compounds 1-2). These small changes are consistent with the similar diol binding affinities exhibited by the $Z$ isomers of $\mathbf{1 - 3}$ in equilibrium competition binding experiments. Additionally, the boronic esters maintain conjugation with the aryl ring $\left(0.6^{\circ} \leq \psi \leq 20^{\circ}\right)$ after binding EG. This favorable conformation, combined with the absence of repulsive interactions with the ortho groups, likely underlies the improved binding of $Z$ azobenzene boronic acids compared to their $E$ isomers.
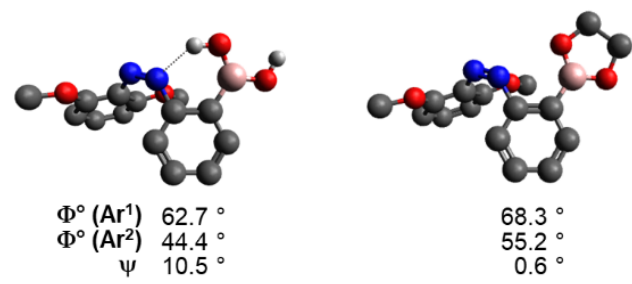

Figure 7. Calculated structure of (Z)-3 before and after binding EG.

Based on the calculated structures, we designed control compounds to experimentally demonstrate that the high $K_{\text {rel }}$ for $\mathbf{3}$ arises from a combination of (i) H-bonding to the azo group, which stabilizes the $(E)$-boronic acid; (ii) additional H-bonding to one of the ortho-methoxy groups, providing further stabilization; and (iii) steric destabilization of the $(E)$-boronic ester. First, we synthesized compound $\mathbf{5}$, in which one of the methoxy substituents was moved from the ortho position to the para position. When $\mathbf{5}$ was subjected to the competition experiment, $K_{\text {rel }}$ was determined to be 2.4 , almost an order of magnitude lower than that of $\mathbf{3}$ (see Figure S15 for ${ }^{1} \mathrm{H}$ NMR). The calculated structures of $\mathbf{5}$ show that the boronic acid and ester both adopt planar conformations. (Figure S33-34). This control experiment suggests that stabilization of the boronic acid by double H-bonding only partially disfavors esterification; steric interactions that destabilize the ester are also required to achieve high $K_{\text {rel }}$.

Next, we synthesized di-ortho-ethyl azobenzene $\mathbf{6}$, which is sterically comparable to compound $\mathbf{3}$, but lacks the ability to participate in additional hydrogen bonding with the ortho positions. When subjected to the competition experiment, $K_{\text {rel }}$ was determined to be only 1.3 (Figure S16). Calculated structure of $\mathbf{6}$ show significant twisting in both the boronic acid $\left(\Phi\left(\mathrm{Ar}^{1}\right)=43^{\circ}\right.$, Figure S35) and boronic ester $\left(\Phi\left(\mathrm{Ar}^{1}\right)=42^{\circ}\right)$. This control experiment demonstrates that steric interactions alone cannot explain the $K_{\text {rel }}$ trend for 1-3; H-bonding to stabilize the boronic acid and planarize the $(E)$ azobenzene are essential.

Lastly, we synthesized stilbene 7, which is sterically comparable to 2 and capable of $E \rightarrow Z$ isomerization but is unable to engage in intramolecular hydrogen bonding (Figure S36). For compound 7, $K_{\text {rel }}$ was determined to be only 1.8 (Figure S17). This modest $K_{\text {rel }}$ value likely arises from steric effects alone. Stilbene 7 also allowed us to experimentally probe the relative effect of intramolecular Hbonding on the thermodynamics of boronic acid esterification for $E$ vs. $Z$ isomers. We performed an equilibrium competition binding experiment between $\mathbf{2}$ and $\mathbf{7}$ and observed that the stilbenes have higher binding affinities for diols compared to their azobenzene analogs. Specifically, $E$ stilbene bound EG 6-fold more than the $E$ azobenzene, whereas the $Z$ stilbene bound EG 2.6-fold more than the $\mathrm{Z}$ azobenzene. While the $\mathrm{O}-\mathrm{H} \cdots \mathrm{N}$ H-bond is present in both $E$ and $Z$ azobenzene isomers, its stabilizing influence is more significant in the $E$ isomer; furthermore, the $E$ isomer presents multiple H-bond acceptors.

In summary, calculated structures, supported by experimental results with control compounds 5-7, lead us to the following conclusions:

1) Stabilization of boronic acids by adjacent H-bond acceptors can disfavor esterification. This effect is further exacerbated by the presence of multiple H-bond acceptors, as in the case of $\mathbf{3}$ and, to a lesser extent, $\mathbf{2}$, in which both the proximal azo $N$ and the ortho groups of the distal arene act as $\mathrm{H}$-bond acceptors. 
2) In $E$ azobenzenes, the degree of twisting away from the planar conformation imposed by steric interactions offers an additional synthetic handle to tune the stability of dynamic covalent bonds. Previous approaches have considered the effect of photoswitch conjugation on its propensity to form a dynamic bond; ${ }^{30}$ our work suggests a complementary design principle, in which the structure of the dynamic bond can also affect the stabilization of the photoswitch by conjugation.

3) The twisted structure of $Z$ azobenzenes requires fewer conformational changes to accommodate boronic acid esterification, and also precludes additional H-bonding interactions. Thus, esterification is more favorable for the $Z$ isomer than for the $E$ isomer.

While previous studies have probed the structural features that affect the thermodynamics of the boronic acid-ester dynamic bond in detail, they have generally focused on parameters such as the electronics of the boronic acid, the presence of proximal basic amines, or the diol structure. ${ }^{39,41-42,44,58}$ The insights gained from our studies provide a framework for future designs of boronic acids where proximal functional groups can enhance or disrupt the stability of the dynamic bond.

\section{Diol binding can be tuned through irradiation with different wavelengths of light}

We selected 3 as the optimal photoswitch to tune diol binding because of its large $K_{\text {rel }}$ and favorable photochemical properties. We envisioned that the ratio of the $E$ and $Z$ isomers should dictate the overall boronic acid-ester equilibrium, wherein the amount of bound diol should increase as the ratio of $(Z)-\mathbf{3}$ to $(E)-\mathbf{3}$ increases. As the PSS $(\% Z)$ is wavelength dependent, the ratio of $Z$ to $E$ can be tuned by irradiation with different wavelengths of light. We prepared a solution of $(E)-3$ and EG (1:1) in DMSO- $d_{6}$, in which the

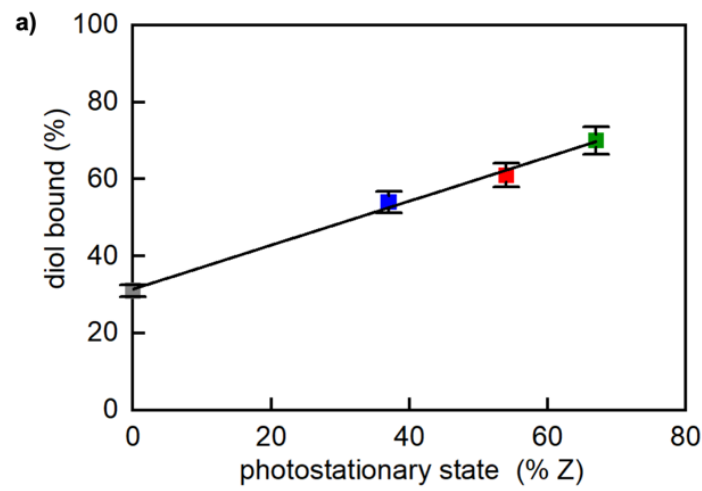

b)

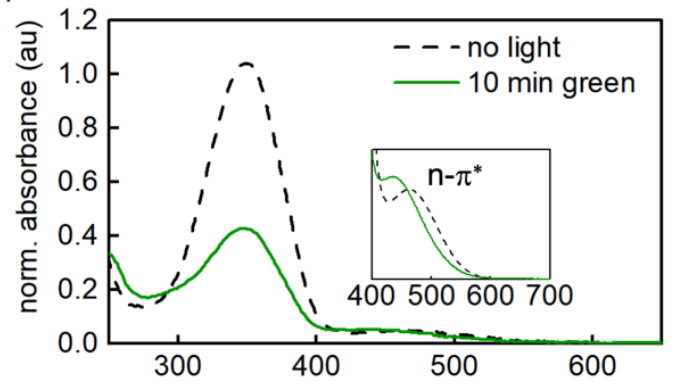

Figure 8. (a) Percent diol binding as a function of PSS, which is achieved thermally (gray) or with blue, red, or green light. Error bars represent integration error, and the line is the least squares fit. (b) UV-Vis profile of $\mathbf{3}$ in acetonitrile before (black) and after (green) irradiation with green LEDs for 10 minutes. azobenzene bound $30 \%$ of the available EG prior to irradiation (Figure 8a, see Figure S19 for ${ }^{1} \mathrm{H}$ NMR spectrum). Irradiating the sample with blue, red, or green irradiation yielded different photostationary states, and higher photostationary states correlated linearly with increased diol binding. Up to $70 \%$ of the diol could be bound with green light. The high barrier for $Z \rightarrow E$ thermal isomerization ensures that the resulting equilibrium is maintained for the duration of the experiment ( 24 hours), such that continuous irradiation is not required. By irradiating with a wavelength of light corresponding to a lower PSS, the equilibrium can be shifted to favor boronic acid and enables release of diol into solution. Figure $8 \mathrm{~b}$ shows the UV-Vis absorbance spectrum of $\mathbf{3}$ before and after irradiation with green light. The ability to achieve high PSS for both $E \rightarrow Z$ and $Z \rightarrow E$ isomerization arises from the separation of the n$\pi^{*}$ transitions of the $E$ and $Z$ isomers (Figure 8b, inset).

\section{Photoswitching modulates boronic ester equilibria with different diols}

Next, we investigated the generality of photoswitchable binding to boronic acid 3 with different diols. Using $\mathbf{3}$, we observed $K_{\text {rel }}$ values between 6 and 20 for a variety of diols, including a 1,3-diol (8), simple cyclic diols (9-10), nitro-catechol (11), and a variety of complex and biologically relevant diols, including glucose (14), adenosine (15), and the chemotherapeutic drug capecitabine (16) (Figure 9). Phenylboronic acid-containing polycarbonates have previously been used for the $\mathrm{pH}$-dependent release of diols including capecitabine. ${ }^{59}$ We envision $\mathbf{3}$ could provide a complementary light-driven strategy. Diols of interest could first be bound in the $Z$ state, where release could be controlled by isomerization to the $E$ isomer.

We qualitatively observed that some diols bound 3 poorly in the standard NMR assay conditions $\left(<1 \% \mathrm{D}_{2} \mathrm{O}\right.$ in DMSO- $\left.d_{6}\right)$; when exogenous water was added to the 4-nitrocatechol (9) equilibrium competition binding experiment, we exclusively observed only boronic acid for both isomers. In contrast, some cyclic diols and polyols (such as cis-1,2-cylcopentanediol (9) and gluconolactone derivative (13)) have higher binding constants, and additional exogenous water $\left(6 \%\right.$ in DMSO- $\left.d_{6}\right)$ was needed in the competition experiment to observe any unbound acid, consistent with previous studies using simple arylboronic acids. ${ }^{41}$ These results demonstrate that while the relative binding affinities between $Z$ and $E$ are

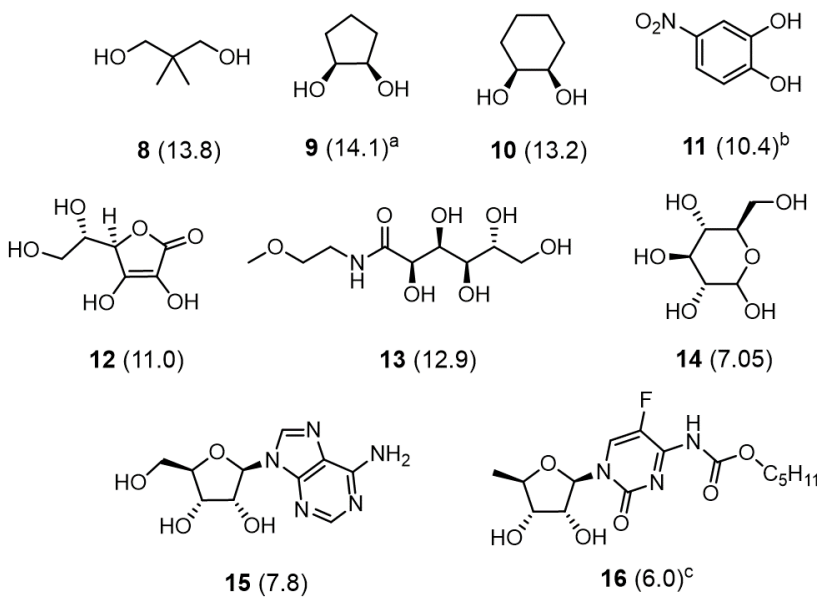

Figure 9. $K_{\text {rel }}$ (reported in parentheses) between $(Z)-\mathbf{3}$ and $(E)-\mathbf{3}$ with various diols $\left(20 \mathrm{mM}\right.$ in DMSO- $\mathrm{d}_{6}$ with $\left.2 \mu \mathrm{L} \mathrm{D}_{2} \mathrm{O}\right)$. Standard deviations were between 0.1 and 2.4 and are reported in Figures S20 through $\mathrm{S} 28$. ${ }^{\text {aExcess }} \mathrm{D}_{2} \mathrm{O}$ was necessary due to high binding affinity. ${ }^{\text {}} \mathrm{No}$ additional $\mathrm{D}_{2} \mathrm{O}$ was used due to low binding affinity. ${ }^{c}$ Lower concentration $(8 \mathrm{mM})$ was used due to sample requirements. 
similar across a range of diols, the absolute equilibrium constants are highly dependent on diol structure. For applications of $\mathbf{3}$ in an aqueous environment, cyclic diols such as $\mathbf{9}$ or polyols such as $\mathbf{1 3}$ will be optimal.

\section{Azobenzene boronic acids reversibly bind diols in fully aqueous environments}

To overcome the low aqueous solubility of small molecule 3 , we designed and synthesized a carboxylate analog of 3, SI-11, and coupled it to amine-terminated 4-arm poly(ethylene glycol) (PEG, $\mathrm{M}_{\mathrm{w}}$ $5 \mathrm{~kg} / \mathrm{mol}$ ) (polymer P1, Figure 10a; see SI for details). While installing the electron-withdrawing ester shortens the thermal halflife of the $Z$ azobenzene to 2.2 days by creating push-pull character (Figure S7), it increases the PSS (\%Z). SI-10, the methyl ester of compound SI-11, reaches $76 \% Z$ after red light irradiation, and returns to $29 \% Z$ after blue light irradiation (Table S4).

We also synthesized a coumarin-tagged gluconolactone derivative (compound 17) to provide a fluorescent output for reversible binding. The gluconolactone tag was chosen based on the performance of 13 in the presence of excess water, suggesting esterification would be favorable in an aqueous environment. The ratio of bound 17 should be increased after irradiation to predominantly $Z$ isomer with red light and decreased after irradiation to predominantly $E$ isomer with blue light. We mixed the boronic acid polymer $\mathbf{P 1}$ and compound $\mathbf{1 7}$ in a 1:4 molar ratio (1:1 boronic acid:17) in a phosphate buffered saline (PBS) solution at $\mathrm{pH}$ 7.5. To analyze the amount of unbound $\mathbf{1 7}$, we filtered the mixture with a $3.0 \mathrm{kDa}$ molecular weight cut-off (MWCO) centrifugal filter such that only free fluorophore (MW $383 \mathrm{~g} / \mathrm{mol}$ ) can pass through the membrane.

a)

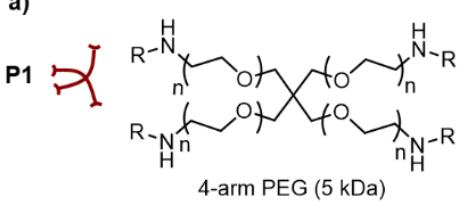

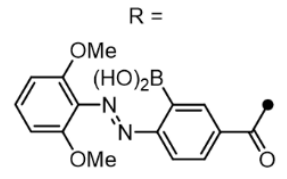

17

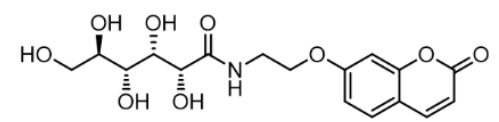

b)
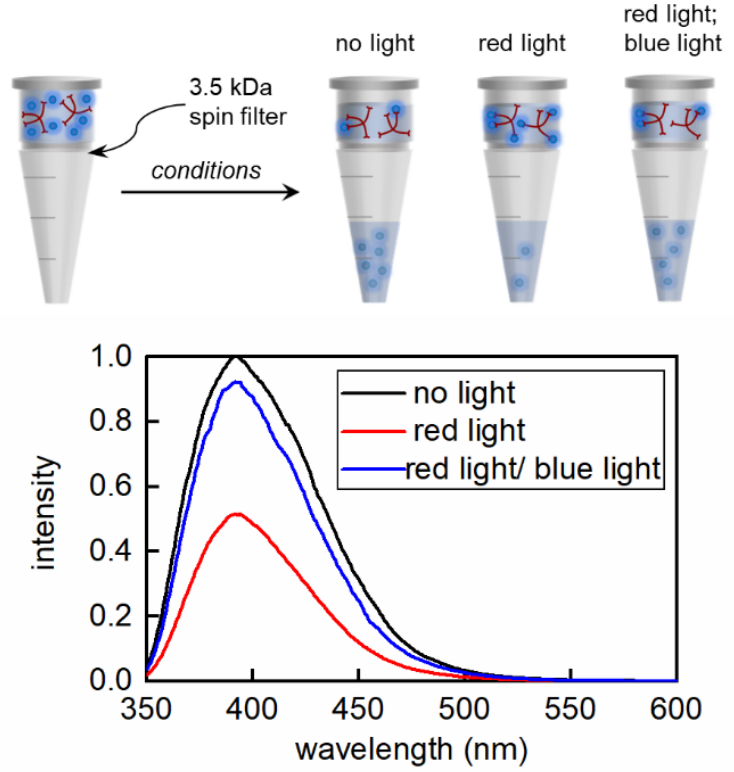

Figure 10. (a) Structures of P1 and gluconolactone-tagged coumarin 17. (b) Illustration of experimental setup and fluorescence spectra of eluents after irradiating and spin filtering each solution.
We next performed UV-vis and fluorescence spectroscopy of the resulting dialysate. Without any irradiation, we observed strong fluorescence in the dialysate indicating that unbound $\mathbf{1 7}$ had passed through the filter (Figure 10b). When the solution was irradiated with red light for 60 minutes before spin filtering, we observed decreased emission, indicating more fluorophore was bound to P1 and unable to pass through the filter. In an analogous experiment, we first irradiated the mixture with red light for 60 minutes, then with blue light for 10 minutes, before centrifugal filtration. In this case, the fluorescence of the dialysate increased to nearly the same level as the no-irradiation control, demonstrating that the fluorophore bound to red-irradiated P1 was released by blue light.

\section{Reversible changes in binding affinity lead to reversible stiffening of hydrogels}

Lastly, we sought to translate the reversible changes in binding affinity to control crosslink density in a hydrogel network. Previously, we demonstrated that reversible changes in binding affinity could lead to sol-gel transitions in a boronic ester crosslinked poly(ethylene glycol) hydrogel, with stiffening occurring with UV or green light and softening occurring with blue light. Based on the photochemical properties of compound $\mathbf{3}$, we reasoned that its incorporation as a crosslink into hydrogels would enable stiffening with red light. We prepared diol-terminated polymer $\mathbf{P 2}$ by ringopening glucono- $\delta$-lactone with amine- terminated 4-arm poly(ethylene glycol) $\left(M_{\mathrm{w}}=5 \mathrm{kDa}\right)$, according to a previous literature procedure $^{32}$. When $\mathbf{P 1}$ and $\mathbf{P 2}$ were mixed in a $1: 1$ ratio in $0.1 \mathrm{M}$ phosphate-buffered saline at $\mathrm{pH} 7.5(10 \mathrm{w} / \mathrm{v} \%)$, a sol was observed, according to the flow-inversion method. Irradiation with red LEDs $(626 \mathrm{~nm})$ for 3 hours promotes the $E \rightarrow Z$ isomerization of the terminal azobenzene boronic acids and leads to the gelation of the mixture. Irradiating this gel for 5 minutes with blue LEDs promotes $Z \rightarrow E$ isomerization and returns the mixture to the sol state (Figure 11a). By alternating irradiation with red and blue light, we could continue to cycle the gel between sol and gel states. Addition of excess free diol $\mathbf{1 3}$ leads to the dissolution of the network, presumably by outcompeting $\mathbf{P 2}$ and disrupting the boronic ester crosslinks.

Toward our lab's effort to design photoreversible matrices for $3 \mathrm{D}$ cell culture, we prepared a hydrogel with $\mathbf{P 1}$ and $\mathbf{P 2}(10 \mathrm{w} / \mathrm{v} \%)$ in Dulbecco's Modified Eagle Medium (DMEM), a common growth medium for a variety of cell types. While the glucose, amino acids, or vitamins in DMEM could interfere with the boronic ester dynamic bond, we were gratified to observe that gelation can still occur in this medium. Bulk mechanical characterization of this hydrogel was performed by oscillatory photorheology at constant strain in the linear viscoelastic region. We first investigated the frequency-dependent properties of this hydrogel from 100 to $0.1 \mathrm{rad} / \mathrm{s}$, which yields information on the dynamics of the crosslinks. The storage modulus (G') represents the material's ability to store energy elastically like a solid, while the loss modulus (G") represents the material's able to dissipate energy into the surrounding environment like a liquid. In materials that behave as elastic solids, such as permanently crosslinked hydrogels, G' and G" are frequency independent. Viscoelastic materials display both solid- and liquidlike behaviors, which depend upon the frequency of the deformation. At high frequencies $(>1 \mathrm{rad} / \mathrm{s})$, where deformation is faster than the dynamic bond exchange, the $\mathbf{P 1} / \mathbf{P 2}$ gel has solid-like behavior, wherein G'> G', The crossover point (where G' = G') occurs at $0.5 \mathrm{rad} / \mathrm{s}$, and below this frequency the gel flows like a liquid due to the reversible hydrolysis of boronic ester crosslinks (Figure 11b). Interestingly, this hydrogel does not undergo sol-gel transitions by the flow inversion method, which are observed when PBS is the solvent. We hypothesize that the additives in DMEM reduce the rate of exchange of the dynamic crosslinks, shifting the crossover point to lower frequencies. The relationship between molecular kinetics and the crossover frequency is the subject of ongoing studies in our laboratory. 
a)
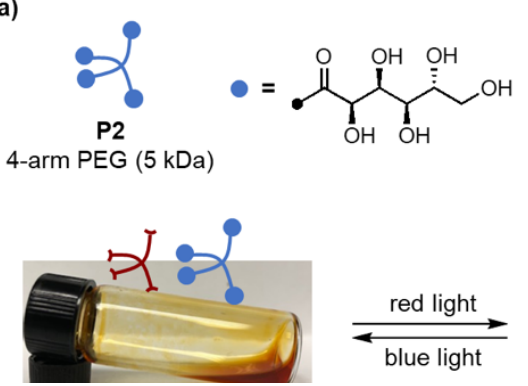
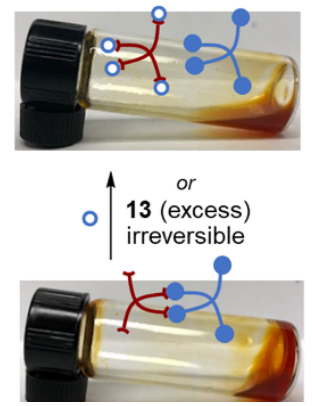

b)

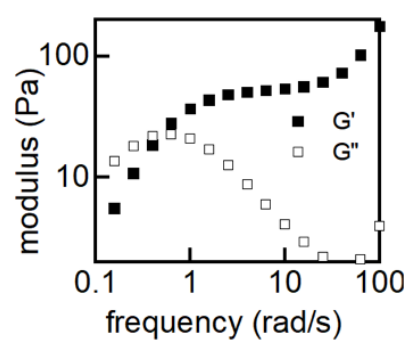

c)

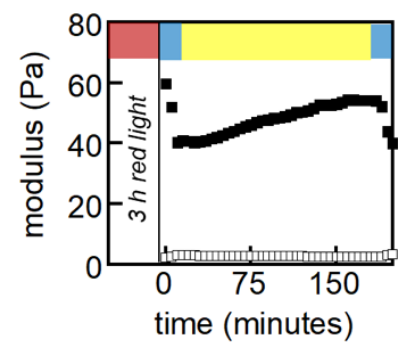

Figure 11. (a) Structure of $\mathbf{P} 2$ and reversible gelation of $\mathbf{P 1}$ and $\mathbf{P 2}$ with red and blue light (10 w/v\%, PBS pH 7.5). (b) Frequency sweep of $\mathbf{P 1}$ and $\mathbf{P 2}$ in DMEM (10 w/v\%, 10\% strain) after stiffening with red light for 3 hours. (c) Reversible mechanical response of $\mathbf{P 1}$ and $\mathbf{P 2}$ in DMEM (10 w/v\%, 10\% strain, $25 \mathrm{rad} / \mathrm{s})$ to different wavelengths of visible light.

The hydrogel in DMEM also demonstrates photodependent changes in stiffness (Figure 11c). The gel was stiffened for three hours with red light, yielding a G' of $60 \mathrm{~Pa}$. Irradiation for 10 minutes with blue light led to a softer gel, decreasing $G^{\prime}$ by $33 \%$. The gel could be stiffened again with yellow light irradiation, increasing G' to $54 \mathrm{~Pa}$ after 3 hours. We propose that the photo-dependent changes in stiffness arise from changes in the hydrogel crosslink density. We hypothesize that the softness of these gels, compared to those previously reported in the literature, ${ }^{31-32,47,60}$ is due to the unusually low binding of the $E$ azobenzene. While these hydrogels may be practical as tools for studying soft tissue mechanics, we envision that increasing the binding constant of the $Z$ isomer while maintaining high $K_{\text {rel }}$ will enable larger ranges of photocontrolled stiffness.

\section{CONCLUSIONS}

We have shown how multiple wavelengths of light can be used to tune the equilibrium of a dynamic covalent bond by harnessing the photostationary state of a photoswitch. While previous reports of photocontrolled dynamic covalent chemistry turn equilibria on or off, our approach offers the ability to modulate the boronic acid/ester equilibrium depending upon the isomerism of an azobenzene photoswitch. The binding equilibrium becomes more favorable as the percentage of $Z$ isomer is increased. Moreover, we show that the range of equilibrium binding constants achieved by a single photoswitch is highly dependent upon ortho, ortho-substitution of the distal ring. In addition to increasing the difference in $E$ and $Z$ binding constants, these ortho substituents impart improved photochemical properties including longer thermal half-lives, increased photostationary states, and visible light photoswitching, making them promising for biomedical applications. The optimized azobenzene identified in these studies can reversibly bind a range of biologically relevant diols. We further demonstrated the application of photoswitchable equilibria to a dynamic covalent hydrogel that can be stiffened with red light. Tuning equilibria with photoswitches represents a powerful approach to noninvasively control the interaction between molecular and macromolecular species. While this work applies this concept to boronic acid-ester exchange, the underlying principles may be used to impart photocontrol on other dynamic reactions, such as imine exchange or thiaMichael addition.

\section{ASSOCIATED CONTENT}

\section{Supporting Information}

Synthetic procedures; characterization data for new compounds; UV-vis spectra; Arrenhius plots; ${ }^{1} \mathrm{H}$ NMR for competition experiments; XYZ coordinates of calculated structures; single-crystal Xray data; rheological data.

\section{AUTHOR INFORMATION}

\section{Corresponding Author}

jkalow@northwestern.edu

\section{Present Addresses}

$\dagger$ Department of Chemistry and Biochemistry, Montana State University, Bozeman, Montana 59717, United States.

\section{Notes}

J. A. K. and J. V. A. have filed a provisional patent application (U.S. Prov. 62/673,312).

\section{ACKNOWLEDGMENT}

This material is based upon work supported by the National Science Foundation under CAREER CHE-1847948 and a 3M NonTenured Faculty Award (J.A.K.). The authors thank the Dichtel Laboratory for use of their fluorimeter, as well as Jacob Ishibashi, Pradipta Das, Boyeong Kang, and Christopher Eckdahl for helpful comments. This work made use of NMR and MS instrumentation at the Integrated Molecular Structure Education and Research Center (IMSERC) at Northwestern, which has received support from the NSF (NSF CHE-9871268); Soft and Hybrid Nanotechnology Experimental (SHyNE) Resource (NSF ECCS-1542205); the State of Illinois and International Institute for Nanotechnology. This research was supported in part through the computational resources and staff contributions provided for the Quest high performance computing facility at Northwestern University, which is jointly supported by the Office of the Provost, the Office for Research, and Northwestern University Information Technology. Rheological measurements were performed at the MatCI Facility, which receives support from the MRSEC Program (NSF DMR-1720139) of the Materials Research Center at Northwestern University.

\section{REFERENCES}

1. Jin, Y. H.; Yu, C.; Denman, R. J.; Zhang, W., Recent advances in dynamic covalent chemistry. Chem. Soc. Rev. 2013, 42, 6634-6654.

2. Winne, J. M.; Leibler, L.; Du Prez, F. E., Dynamic covalent chemistry in polymer networks: a mechanistic perspective. Polym. Chem. 2019, 10, 6091-6108.

3. Zhang, W.; Jin, Y., Dynamic covalent chemistry : principles, reactions, and applications. First edition. ed.; Wiley, Hoboken, NJ, 2017; p. 1 online resource.

4. Wojtecki, R. J.; Meador, M. A.; Rowan, S. J., Using the dynamic bond to access macroscopically responsive structurally dynamic polymers. Nat. Mater. 2011, 10, 14-27.

5. Boehnke, N.; Cam, C.; Bat, E.; Segura, T.; Maynard, H. D., Imine Hydrogels with Tunable Degradability for Tissue Engineering. Biomacromolecules 2015, 16, 2101-2108.

6. Lohse, M. S.; Bein, T., Covalent Organic Frameworks: Structures, Synthesis, and Applications. Adv. Funct. Mater. 2018, 28,1705553 
7. Liu, Y.; Lehn, J. M.; Hirsch, A. K. H., Molecular Biodynamers: Dynamic Covalent Analogues of Biopolymers. Acc. Chem. Res. 2017, 50, 376-386.

8. Rottger, M.; Domenech, T.; van der Weegen, R.; Nicolay, A. B. R.; Leibler, L., High-performance vitrimers from commodity thermoplastics through dioxaborolane metathesis. Science 2017, 356, 62-65.

9. Gu, R. R.; Flidrova, K.; Lehn, J. M., Dynamic Covalent Metathesis in the $\mathrm{C}=\mathrm{C} / \mathrm{C}=\mathrm{N}$ Exchange between Knoevenagel Compounds and Imines. J. Am. Chem. Soc. 2018, 140, 5560-5568.

10. Maeda, T.; Otsuka, H.; Takahara, A., Dynamic covalent polymers: Reorganizable polymers with dynamic covalent bonds. Prog. Polym. Sci. 2009, 34, 581-604.

11. Rapp, T. L.; DeForest, C. A., Visible Light-Responsive Dynamic Biomaterials: Going Deeper and Triggering More. Adv. Healthc. Mater. 2020.

12. Scott, T. F.; Schneider, A. D.; Cook, W. D.; Bowman, C. N., Photoinduced plasticity in cross-linked polymers. Science 2005, 308, 1615-1617.

13. Worrell, B. T.; McBride, M. K.; Lyon, G. B.; Cox, L. M.; Wang, C.; Mavila, S.; Lim, C. H.; Coley, H. M.; Musgrave, C. B.; Ding, Y.; Bowman, C. N., Bistable and photoswitchable states of matter. Nat. Commun. 2018, 9, 2804.

14. Liu, J. H.; Butt, H. J.; Wu, S., Reconfigurable Surfaces Based on Photocontrolled Dynamic Bonds. Adv. Funct. Mater. 2019, 30, 1907605

15. Gong, T.; Feng, J. C.; Wei, W.; Huang, W., Recent progress in diarylethene as a photoswitching unit. Prog. Chem. 2006, 18, 698-706.

16. Bandara, H. M. D.; Burdette, S. C., Photoisomerization in different classes of azobenzene. Chem. Soc. Rev. 2012, 41, 18091825.

17. Helmy, S.; Leibfarth, F. A.; Oh, S.; Poelma, J. E.; Hawker, C. J.; de Alaniz, J. R., Photoswitching Using Visible Light: A New Class of Organic Photochromic Molecules. J. Am. Chem. Soc. 2014, 136, 8169-8172.

18. Klajn, R., Spiropyran-based dynamic materials. Chem. Soc. Rev. 2014, 43, 148-184.

19. van Dijken, D. J.; Kovaricek, P.; Ihrig, S. P.; Hecht, S., Acylhydrazones as Widely Tunable Photoswitches. J. Am. Chem. Soc. 2015, 137, 14982-14991.

20. Gerwien, A.; Reinhardt, T.; Mayer, P.; Dube, H., Synthesis of Double-Bond-Substituted Hemithioindigo Photoswitches. Org. Lett. 2018, 20, 232-235.

21. Kortekaas, L.; Browne, W. R., The evolution of spiropyran: fundamentals and progress of an extraordinarily versatile photochrome. Chem. Soc. Rev. 2019, 48, 3406-3424.

22. Liu, D.; Sponza, A. D.; Yang, D. D.; Chiu, M., Modulating Polymer Dispersity with Light: Cationic Polymerization of Vinyl Ethers Using Photochromic Initiators. Angew. Chem. Int. Ed. 2019, 58, 16210-16216.

23. Lvov, A. G.; Yadykov, A. V.; Lyssenko, K. A.; Heinemann, F. W.; Shirinian, V. Z.; Khusniyarov, M. M., Reversible Shifting of a Chemical Equilibrium by Light: The Case of Keto-Enol Tautomerism of a beta-Ketoester. Org. Lett. 2020, 22 , 604-609.

24. Kathan, M.; Hecht, S., Photoswitchable molecules as key ingredients to drive systems away from the global thermodynamic minimum. Chem. Soc. Rev. 2017, 46, 5536-5550.

25. Lemieux, V.; Gauthier, S.; Branda, N. R., Selective and sequential photorelease using molecular switches. Angew. Chem. Int. Ed. 2006, 45, 6820-6824.

26. Gostl, R.; Hecht, S., Photoreversible Prodrugs and Protags: Switching the Release of Maleimides by Using Light under Physiological Conditions. Chem. Eur. J. 2015, 21, 44224427.

27. Fuhrmann, A.; Gostl, R.; Wendt, R.; Kotteritzsch, J.; Hager, M. D.; Schubert, U. S.; Brademann-Jock, K.; Thunemann,
A. F.; Nochel, U.; Behl, M.; Hecht, S., Conditional repair by locally switching the thermal healing capability of dynamic covalent polymers with light. Nat. Commun. 2016, 7. 13623

28. Asadirad, A. M.; Boutault, S.; Erno, Z.; Branda, N. R., Controlling a Polymer Adhesive Using Light and a Molecular Switch. J. Am. Chem. Soc. 2014, 136, 3024-3027.

29. Kathan, M.; Eisenreich, F.; Jurissek, C.; Dallmann, A.; Gurke, J.; Hecht, S., Light-driven molecular trap enables bidirectional manipulation of dynamic covalent systems. Nat. Chem. 2018, 10, 1031-1036.

30. Kathan, M.; Kovaříček, P.; Jurissek, C.; Senf, A.; Dallmann, A.; Thünemann, A. F.; Hecht, S., Control of Imine Exchange Kinetics with Photoswitches to Modulate Self-Healing in Polysiloxane Networks by Light Illumination. Angew. Chem. Int. Ed. 2016, 55, 13882-13886.

31. Yesilyurt, V.; Webber, M. J.; Appel, E. A.; Godwin, C.; Langer, R.; Anderson, D. G., Injectable Self-Healing GlucoseResponsive Hydrogels with $\mathrm{pH}-$ Regulated Mechanical Properties. Adv. Mater. 2016, 28, 86-91.

32. Yesilyurt, V.; Ayoob, A. M.; Appel, E. A.; Borenstein, J. T.; Langer, R.; Anderson, D. G., Mixed Reversible Covalent Crosslink Kinetics Enable Precise, Hierarchical Mechanical Tuning of Hydrogel Networks. Adv. Mater. 2017, 29, 1605947.

33. Guan, Y.; Zhang, Y., Boronic acid-containing hydrogels: synthesis and their applications. Chem. Soc. Rev. 2013, 42, 81068121.

34. Cromwell, O. R.; Chung, J.; Guan, Z. B., Malleable and Self-Healing Covalent Polymer Networks through Tunable Dynamic Boronic Ester Bonds. J. Am. Chem. Soc. 2015, 137, 64926495.

35. Hargrove, A. E.; Reyes, R. N.; Riddington, I.; Anslyn, E. V.; Sessler, J. L., Boronic Acid Porphyrin Receptor for Ginsenoside Sensing. Org. Lett. 2010, 12, 4804-4807.

36. Ni, N. T.; Laughlin, S.; Wang, Y. J.; Feng, Y.; Zheng, Y. J.; Wang, B. H., Probing the general time scale question of boronic acid binding with sugars in aqueous solution at physiological $\mathrm{pH}$. Bioorg. Med. Chem. 2012, 20, 2957-2961.

37. Chapin, B. M.; Metola, P.; Vankayala, S. L.; Woodcock, H. L.; Mooibroek, T. J.; Lynch, V. M.; Larkin, J. D.; Anslyn, E. V., Disaggregation is a Mechanism for Emission Turn-On of orthoAminomethylphenylboronic Acid-Based Saccharide Sensors. $J$. Am. Chem. Soc. 2017, 139, 5568-5578.

38. Sun, X. L.; James, T. D.; Anslyn, E. V., Arresting "Loose Bolt" Internal Conversion from $-\mathrm{B}(\mathrm{OH})(2)$ Groups is the Mechanism for Emission Turn-On in orthoAminomethylphenylboronic Acid-Based Saccharide Sensors. J. Am. Chem. Soc. 2018, 140, 2348-2354.

39. Sun, X. L.; Chapin, B. M.; Metola, P.; Collins, B.; Wang, B. H.; James, T. D.; Anslyn, E. V., The mechanisms of boronate ester formation and fluorescent turn-on in orthoaminomethylphenylboronic acids. Nat. Chem. 2019, 11, 768-778.

40. Monajemi, H.; Cheah, M. H.; Lee, V. S.; Zain, S. M.; Abdullah, W. A. T. W., On the kinetics and reaction mechanisms of boronic acid in interaction with diols for non-enzymatic glucose monitoring applications: a hybrid DFT study. RSC $A d v$. 2014, 4, 10505-10513.

41. Marinaro, W. A.; Prankerd, R.; Kinnari, K.; Stella, V. J., Interaction of Model Aryl- and Alkyl-Boronic Acids and 1,2-Diols in Aqueous Solution. J. Pharm. Sci. 2015, 104, 1399-1408.

42. Brooks, W. L. A.; Deng, C. C.; Sumerlin, B. S., Structure-Reactivity Relationships in Boronic Acid-Diol Complexation. ACS Omega 2018, 3, 17863-17870.

43. Martinez-Aguirre, M. A.; Flores-Alamo, M.; Medrano, F.; Yatsimirsky, A. K., Examination of pinanediol-boronic acid ester formation in aqueous media: relevance to the relative stability of trigonal and tetrahedral boronate esters. Org. Biomol. Chem. 2020, 18, 2716-2726. 
44. Furikado, Y.; Nagahata, T.; Okamoto, T.; Sugaya, T.; Iwatsuki, S.; Inamo, M.; Takagi, H. D.; Odani, A.; Ishihara, K., Universal Reaction Mechanism of Boronic Acids with Diols in Aqueous Solution: Kinetics and the Basic Concept of a Conditional Formation Constant. Chem. Eur. J. 2014, 20, 13194-13202.

45. Kano, N.; Yoshino, J.; Kawashima, T., Photoswitching of the Lewis Acidity of a Catecholborane Bearing an Azo Group Based on the Change in Coordination Number of Boron. Org. Lett. 2005, 7, 3909-3911.

46. Yoshino, J.; Kano, N.; Kawashima, T., Synthesis of organoboron compounds bearing an azo group and substituent effects on their structures and photoisomerization. Tetrahedron 2008, 64, 7774-7781.

47. Accardo, J. V.; Kalow, J. A., Reversibly tuning hydrogel stiffness through photocontrolled dynamic covalent crosslinks. Chem. Sci. 2018, 9, 5987-5993.

48. Bahrenburg, J.; Rottger, K.; Siewertsen, R.; Renth, F.; Temps, F., Sequential photoisomerisation dynamics of the pushpull azobenzene Disperse Red 1. Photochem. Photobiol. Sci. 2012, $11,1210-1219$.

49. Bléger, D.; Schwarz, J.; Brouwer, A. M.; Hecht, S., oFluoroazobenzenes as Readily Synthesized Photoswitches Offering Nearly Quantitative Two-Way Isomerization with Visible Light. J. Am. Chem. Soc. 2012, 134, 20597-20600.

50. Samanta, S.; McCormick, T. M.; Schmidt, S. K.; Seferos, D. S.; Woolley, G. A., Robust visible light photoswitching with ortho-thiol substituted azobenzenes. Chem. Commun. 2013, 49, 10314-10316.

51. Knie, C.; Utecht, M.; Zhao, F.; Kulla, H.; Kovalenko, S.; Brouwer, A. M.; Saalfrank, P.; Hecht, S.; Bléger, D., orthoFluoroazobenzenes: Visible Light Switches with Very LongLivedZIsomers. Chem. Eur. J. 2014, 20, 16492-16501.
52. Dong, M. X.; Babalhavaeji, A.; Samanta, S.; Beharry, A. A.; Woolley, G. A., Red-Shifting Azobenzene Photoswitches for in Vivo Use. Acc. Chem. Res. 2015, 48, 2662-2670.

53. Ahmed, Z.; Siiskonen, A.; Virkki, M.; Priimagi, A., Controlling azobenzene photoswitching through combined orthofluorination and -amination. Chem. Commun. 2017, 53, 1252012523.

54. Dong, M.; Babalhavaeji, A.; Collins, C. V.; Jarrah, K.; Sadovski, O.; Dai, Q. Y.; Woolley, G. A., Near-Infrared Photoswitching of Azobenzenes under Physiological Conditions. $J$. Am. Chem. Soc. 2017, 139, 13483-13486.

55. Konrad, D. B.; Savasci, G.; Allmendinger, L.; Trauner, D.; Ochsenfeld, C.; Ali, A. M., Computational Design and Synthesis of a Deeply Red-Shifted and Bistable Azobenzene. $J$. Am. Chem. Soc. 2020, 142, 6538-6547.

56. Schneider, H. J., Hydrogen bonds with fluorine. Studies in solution, in gas phase and by computations, conflicting conclusions from crystallographic analyses. Chem. Sci. 2012, 3, 1381-1394.

57. Taylor, R., The hydrogen bond between N-H or O-H and organic fluorine: favourable yes, competitive no. Acta Cryst. B 2017, 73, 474-488.

58. Collins, B. E.; Metola, P.; Anslyn, E. V., On the rate of boronate ester formation in ortho-aminomethyl-functionalised phenyl boronic acids. Supramol. Chem. 2013, 25, 79-86.

59. Aguirre-Chagala, Y. E.; Santos, J. L.; Huang, Y. X.; Herrera-Alonso, M., Phenylboronic Acid-Installed Polycarbonates for the $\mathrm{pH}$-Dependent Release of Diol-Containing Molecules. ACS Macro Lett. 2014, 3, 1249-1253.

60. Tang, S. C.; Ma, H.; Tu, H. C.; Wang, H. R.; Lin, P. C.; Anseth, K. S., Adaptable Fast Relaxing Boronate-Based Hydrogels for Probing Cell-Matrix Interactions. Adv. Sci. 2018, 5, 1800638. 


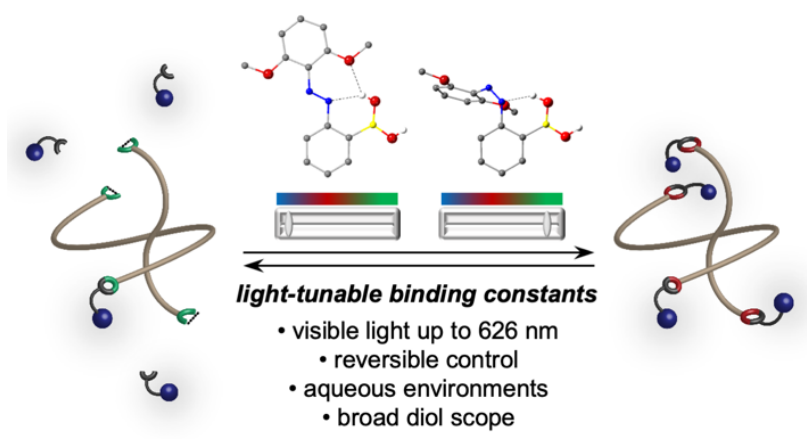

